\title{
Experimental study of the effect of rotation on large amplitude internal waves
}

\author{
R. H.J. Grimshaw, ${ }^{1, \text { a) K. R. Helfrich, }}{ }^{2}$ and E. R. Johnson ${ }^{3}$ \\ 1) Department of Mathematical Sciences, Loughborough University, UK \\ ${ }^{2)}$ Department of Physical Oceanography, Woods Hole Oceanographic Institution, Woods Hole, \\ U.S.A \\ ${ }^{3)}$ Department of Mathematics, University College London, U.K.
}

Large amplitude internal waves are commonly observed in the coastal ocean. In the weakly nonlinear long wave régime, they are often modeled by the Korteweg-de Vries equation, which predicts that the long-time outcome of generic localised initial conditions is a train of internal solitary waves. However, when the effect of background rotation is taken into account, it is known from several theoretical and numerical studies that the formation of solitary waves is inhibited, and instead nonlinear wave packets form. In this paper, we report the results from a laboratory experiment on the Coriolis platform which describes this process.

\section{INTRODUCTION}

Internal solitary waves have been intensively studied over the past few decades, due to the in situ and remote sensing evidence for their common occurrence in coastal and marginal seas, see the reviews by Grimshaw ${ }^{5}$, Holloway et $\mathrm{al}^{11}$ and Helfrich and Melville ${ }^{10}$ for instance. It is now commonly accepted that the canonical model for describing their dynamics is the well-known Korteweg-de Vries (KdV) equation, see (1) below, in which a balance between weak nonlinearity, represented an amplitude parameter $a / h_{0}$, and weak nonhydrostatic linear dispersion, represented by the parameter $h_{0}^{2} / L^{2}$ leads to the existence of solitary waves. Here $a$ is a wave amplitude scale, $h_{0}$ is a suitable depth scale, and $L$ is a wave length scale. $\mathrm{KdV}$ models with extensions to include variable bottom topography, dissipation and other effects have been applied to oceanographic, and to similar atmospheric observations, with generally good results. The essential qualitative features of observed internal solitary waves are well captured by these KdV models, although not always providing precise quantitative information.

However, the significant effects of the earth's rotation have only recently received comparable attention, This is because while the waves are long with respect to the depth, they are short when compared to the internal deformation radius, $L_{R}=c_{0} / f$, that is $L_{R} / L>>1$. Here $c_{0}$ is the linear long wave phase speed, and $f$ is the Coriolis frequency. Consequently, rotational effects have often been ignored. However, although rotational effects are small on the scale of an individual wave, observed waves often survive for several days, and then the influence of background can be significant. Indeed, in a series of recent papers, Helfrich ${ }^{9}$ and Grimshaw and Helfrich ${ }^{6,7}$ have shown that the long-time effect of rotation is the destruction of an internal solitary wave by the radiation of inertia-gravity waves, and its replacement by a coherent steadily propagating nonlinear wave packet.

For internal solitary waves the $\mathrm{KdV}$ equation is, expressed in the reference frame moving with the linear

\footnotetext{
a) Electronic mail: R.H.J.Grimshaw@lboro.ac.uk
}

long wave speed $c_{0}$,

$$
A_{t}+\nu A A_{x}+\lambda A_{x x x}=0 .
$$

Here $A(x, t)$ is the amplitude of the linear long wave mode $\phi(z)$ corresponding to the linear long wave phase speed $c_{0}$, which is determined from the modal equation

$$
\begin{aligned}
& c_{0}^{2}\left\{\rho_{0} \phi_{z}\right\}_{z}-g \rho_{0 z} \phi=0, \quad \text { for } h<z<0, \\
& \text { and } \phi=0 \text { at } z=-h, \quad c_{0}^{2} \phi_{z}=g \phi, \quad \text { at } \quad z=0 \text {.(3) }
\end{aligned}
$$

Here $\rho_{0}(z)$ is the stably stratified background density stratification, and the the coefficients $\nu$ and $\lambda$ are given by

$$
\begin{gathered}
I \nu=3 c_{0}^{2} \int_{-h}^{0} \rho_{0} \phi_{z}^{3} d z, \quad I \lambda=c_{0}^{2} \int_{-h}^{0} \rho_{0} \phi^{2} d z \\
\text { where } \quad I=2 c_{0} \int_{-h}^{0} \rho_{0} \phi_{z}^{2} d z .
\end{gathered}
$$

The solitary wave solution is

$$
A=a_{s} \operatorname{sech}^{2}\left(\frac{x-V t}{D}\right), \quad V=\frac{\nu a_{s}}{3}=\frac{4 \lambda}{D^{2}} .
$$

However, as we have already noted above, observed internal waves waves often exist for several inertial periods, and hence the effects of the Earth's background rotation need to be taken into account. Several numerical experiments of model equations, including the Ostrovsky equation (see equation (6) below), and of fully nonlinear equation systems have shown that background rotation tends to inhibit the production of internal solitary waves, see Helfrich ${ }^{9}$, Grimshaw and Helfrich ${ }^{6,7}$, and Stastna et $\mathrm{al}^{16}$, and the references therein. In particular, Helfrich ${ }^{9}$ found that an initial solitary wave disturbance decayed through the radiation of inertial gravity waves, and eventually an envelope wave packet formed.

The simplest model equation which takes account of background rotation is the Ostrovsky equation, which is an adaptation of the $\mathrm{KdV}$ equation (1) given by, see Ostrovsky $^{13}$, Grimshaw $^{4}$ and Grimshaw et al ${ }^{8}$,

$$
\left\{A_{t}+\nu A A_{x}+\lambda A_{x x x}\right\}_{x}=\gamma A
$$




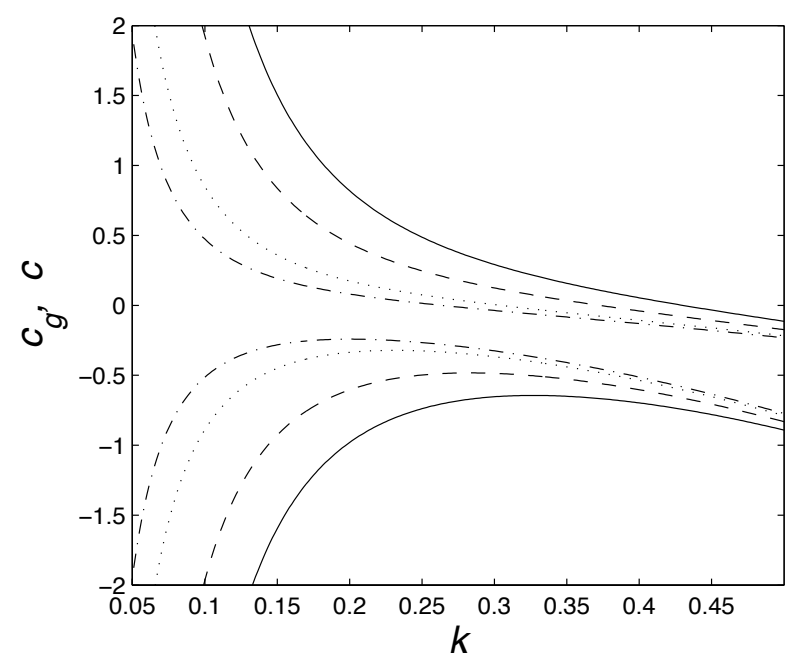

FIG. 1. Plot of the phase velocity (top four curves) and group velocity (bottom four curves) from (8) for the experimental setup and various rotation rates, $T=120,90,60,45 s$ shown as dashdot, dot, dash, solid respectively.

The background rotation is represented by the coefficient $\gamma$ which is given by

$$
\gamma=\frac{f^{2}}{2 c_{0}}
$$

where $f$ is the Coriolis parameter. Once $\rho_{0}(z)$ has been specified, the modal function $\phi(z)$ and the speed $c_{0}$ can be found from (3) and then the coefficients $\nu, \lambda$ evaluated. For oceanic internal and surface waves $\lambda \gamma>0$, see $(4,7)$, and then it is known that equation (6) does not support steady solitary wave solutions, see Grimshaw and Helfrich ${ }^{7}$ and the references therein. Although the additional term on the right-hand side of (6) is a linear long-wave perturbation to the $\mathrm{KdV}$ equation, it has the effect of removing the spectral gap on which solitary waves exist for the KdV equation. Indeed, the linear dispersion relation of the Ostrovsky equation (6) for the phase velocity $c$ and the group velocity $c_{g}$ as a function of wavenumber $k$ are given by

$$
c=\frac{\gamma}{k^{2}}-\lambda k^{2}, \quad c_{g}=\frac{d \omega}{d k}=-\frac{\gamma}{k^{2}}-3 \lambda k^{2} .
$$

Some typical plots are shown figure 1 for coefficients evaluated for our experimental setup (see section 3). For the $\mathrm{KdV}$ equation (1) $(\gamma=0)$ there is a gap in the spectrum for all $c>0$ where solitary waves can exist. But there is no such gap for the Ostrovsky equation, and hence no solitary waves are expected to occur. Complete proofs of the non-existence of solitary waves in the Ostrovsky equation (6) for $\lambda \gamma>0$ have been given by Galkin and Stepanyants ${ }^{2}$, Leonov ${ }^{12}$ and in the simplest form by Grimshaw and Helfrich ${ }^{7}$.

The issue is then the fate of a $\mathrm{KdV}$ solitary wave, when this is set as an initial condition for the Ostrovsky equation (6). This was addressed by Grimshaw and
Helfrich $^{6,7}$ who used a combination of asymptotic analyses and numerical simulations to show that the initial $\mathrm{KdV}$ solitary wave at first decays by radiation into trailing inertia-gravity waves, from which emerges a nonlinear wave packet whose carrier wavenumber is that corresponding to a maximum in the group velocity, and corresponds to the leading disturbance, see figure 1 . The purpose of this paper is to report on a series of laboratory experiments, designed to test the hypothesis that the effect of rotation on an internal solitary wave is to destroy that wave and replace it with a nonlinear wave packet whose envelope propagates steadily with the maximum allowed group velocity. The experiments were conducted in September 2009 using the $13 \mathrm{~m}$ diameter rotating platform at the LEGI-Coriolis Laboratory in Grenoble.

In section 2 we briefly review the theory and report some numerical simulations, which show the emergence of a nonlinear wave packet from an initial solitary wave. Then in section 3 we describe the experimental set-up and outcomes. We conclude in section 4 .

\section{THEORY AND NUMERICAL SIMULATIONS}

\section{A. Linear dispersion and wave packets}

As noted above, $\mathrm{KdV}$ solitary waves are destroyed by the effect of rotation, and a substantial part of the initial energy is converted into a nonlinear wave packet, whose carrier wavenumber is determined by a maximum in the group velocity. For the Ostrovsky equation the group velocity is negative for all wavenumbers $k$, see (8), and has a local maximum $c_{g m}$ where $d c_{g} / d k=0$ at $k=$ $k_{m}$ where $3 \lambda k_{m}^{4}=\gamma$. Note that as $\gamma$ increases so does $k_{m},\left|c_{g m}\right|$. At the group velocity maximum $c_{g m}$, the phase speed $c=c_{p m}$, and

$$
c_{p m}=\frac{2}{3} \sqrt{3 \gamma \lambda}, \quad c_{g m}=-2 \sqrt{3 \gamma \lambda}, \quad k_{m}^{2}=\sqrt{\frac{\gamma}{3 \lambda}} .
$$

In the Appendix, we derive $c_{0}$ and the coefficient $\lambda$ for a two-layer fluid in the Boussinesq approximation. However, while the two-layer model captures the essential dynamics, the continuous stratification of the experiments leads to quantitative differences in the linear dispersion that need to be accounted for when analyzing the experimental results. As shown in section 3 below the experiments have a background stratification that is well-approximated by a hyperbolic tangent profile, see (25). Using this density profile, numerical solutions of the modal equation (3) in the Boussinesq limit where $\rho_{0}=\rho_{1}=$ constant in the first term and $N^{2}=-\left(g / \rho_{1}\right) d \rho_{0} / d z$ gives $c_{0}=6.39 \mathrm{~cm} \mathrm{~s}^{-1}$ for $g^{\prime}=g\left(\rho_{2}-\rho_{1}\right) / \rho_{1}=9.81 \mathrm{~cm} \mathrm{~s}^{-2}$, and the dispersive coefficient of the KdV equation from (4) is $\lambda=231 \mathrm{~cm}^{3} \mathrm{~s}^{-1}$. The corresponding two-layer values are $c_{0}=7 \mathrm{~cm} \mathrm{~s}^{-1}$ and $\lambda=210 \mathrm{~cm}^{3} \mathrm{~s}^{-1}$. The dispersion curves, see (8), for the actual experimental stratification are plotted in 

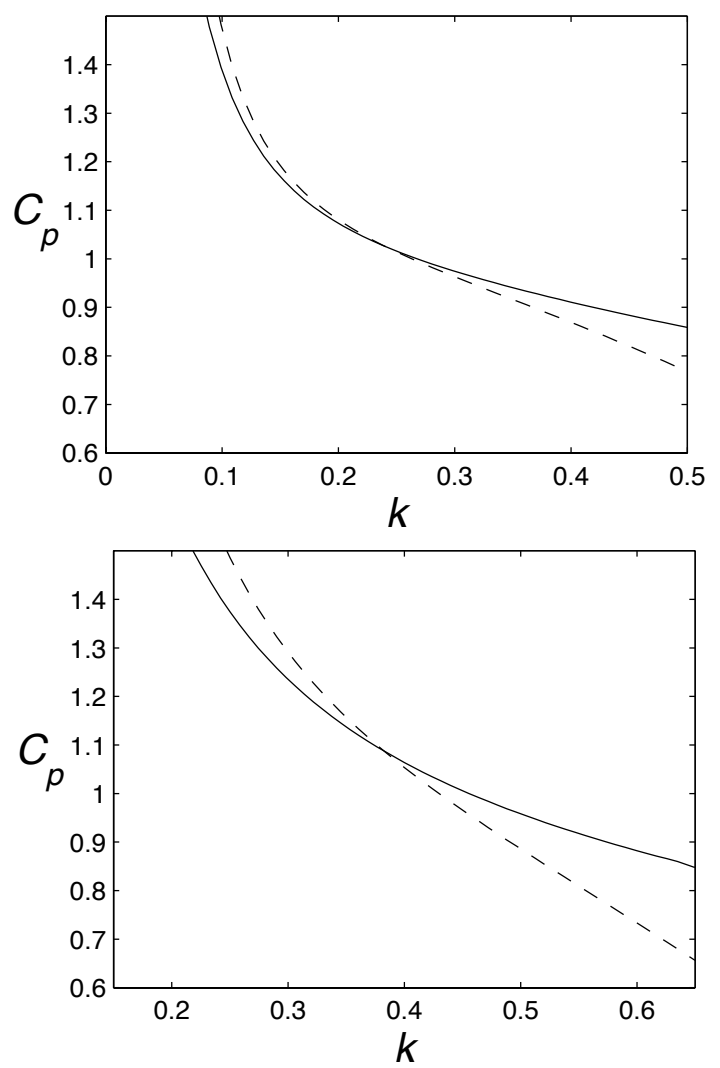

FIG. 2. Plot of the full dispersion relation from solutions of (A12) (solid) and that from the Ostrovsky equation (8) (dashed) for $T=120 \mathrm{~s}$ (left panel) and $T=45 \mathrm{~s}$ (right panel).

figure 1 for several rotation rates. All expressions are non-dimensionalized with a length scale $h_{1}=6 \mathrm{~cm}$ and a velocity scale of $c_{0}=6.39 \mathrm{~cm} \mathrm{~s}^{-1}$.

In the Appendix, we describe the corresponding calculations for the full linear dispersion relation for both the two-layer fluid and the continuously stratified cases. Figure 2 shows a comparison between the full, continuouslystratified dispersion relation from solutions of (A12) and the corresponding dispersion relation (8) from the Ostrovsky equation (with $c_{0}$ added), for the slowest and fastest rotation rates. Figure 3 shows the corresponding plots for the group velocity. The critical points are at $k_{m}=0.20,0.35$ respectively for the full system, whereas the corresponding values for the Ostrovsky equation are $k_{m}=0.19,0.32$ respectively. Note that while there is a good qualitative agreement, we do see some measurable quantitative differences.

Next, we build on the linear dispersion relation to find a weakly nonlinear wave packet. In many physical systems, the canonical model for these is the nonlinear Schrödinger equation (NLS)

$$
i\left(A_{t}+c_{g} A_{x}\right)+\Delta A_{x x}+\mu|A|^{2} A=0,
$$

written for the complex envelope $A(x, t)$ of the weakly
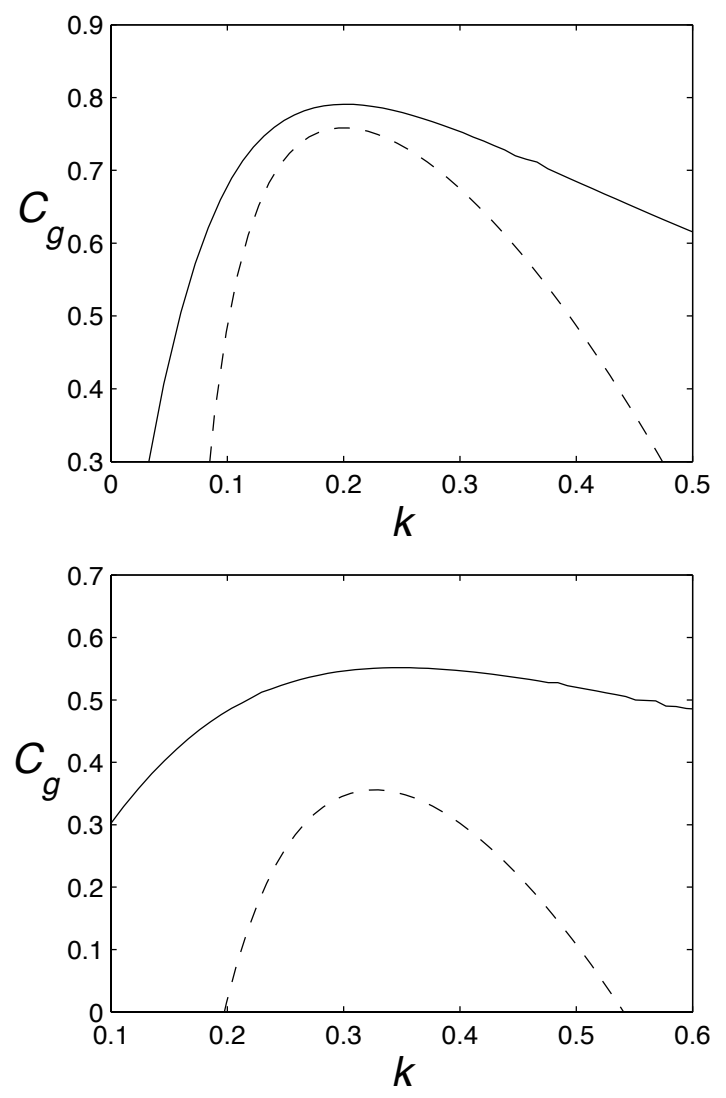

FIG. 3. Plot of the group velocity from the full dispersion relation (solid) and that from the Ostrovsky equation (dashed) for $T=120 \mathrm{~s}$ (left panel) and $T=45 \mathrm{~s}$ (right panel).

nonlinear asymptotic solution,

$$
\eta=A(x, t) \exp (i k x-i \omega t)+\text { c.c. }+\cdots,
$$

where c.c. denotes the complex conjugate. Here $\omega=\omega(k)$ satisfies the linear dispersion relation and at leading order the envelope moves with the linear group velocity $c_{g}=$ $\omega_{k}$. The dispersive term in the NLS equation generically has the coefficient $\Delta=c_{g k} / 2$, but the coefficient $\mu$ of the cubic nonlinear term is system-dependent. Our concern here is precisely with the case when $\Delta=c_{g k} / 2=0$, selecting a wavenumber $k=k_{m}$ where the group velocity has a local extremum.

As described by Grimshaw and Helfrich ${ }^{6,7}$, here the NLS equation is replaced with a higher-order NLS equation

$$
\begin{array}{r}
i\left(A_{t}+c_{g} A_{x}\right)+\Delta A_{x x}+i \delta A_{x x x}+\mu|A|^{2} A \\
+i\left(\alpha|A|^{2} A_{x}+\beta A^{2} A_{x}^{*}\right)=0 .
\end{array}
$$

Here ${ }^{*}$ denotes the complex conjugate. The coefficient of the third-order linear dispersive term is $\delta=-c_{g k k} / 6 \neq 0$, while the coefficients $\alpha, \beta$ of the mixed nonlinear dispersive terms are system-dependent. In the present context, it was shown by Grimshaw and Helfrich ${ }^{6}$ that 
$\delta>0, \mu<0, \alpha>0, \beta>0$ for all wavenumbers $k$. This higher-order NLS equation (12) has the envelope solitary wave solution,

$$
A=F(X-V t) \exp (i \kappa X-i \sigma t), \quad X=x-c_{g} t,
$$

where the gauge $\kappa$ and the chirp $\sigma$ are given by

$$
\begin{gathered}
\mu+2 \kappa \beta=\frac{(\alpha+\beta) \Delta}{3 \delta} \\
\sigma=3 \kappa V+8 \delta \kappa^{3}+\frac{\Delta}{\delta}\left(4 \delta \kappa^{2}-V\right)-\frac{2 \kappa \Delta^{2}}{\delta} \\
\text { and } F(X)=a \operatorname{sech}(K X), \\
\tilde{V}=V+3 \delta \kappa^{2}-\Delta \kappa=\delta K^{2}=\frac{a^{2}(\alpha+\beta)}{6} .
\end{gathered}
$$

This solution requires that $\delta(\alpha+\beta)>0$, which is always the case for the Ostrovsky equation (6), and that $\delta \tilde{V}>0$ which places a constraint on the allowed speeds $V$. It was shown by Grimshaw and Helfrich ${ }^{6}$ that this asymptotic solution can be confirmed numerically, and that it agrees reasonably well with the wave packets found in numerical simulations of the Ostrovsky equation (6).

\section{B. Numerical simulations of the rotation-modified Kadomtsev-Petviashvili equation}

Although our experimental setup was designed to mimic the one-dimensional theory described above as far as possible, the finite transverse width $(5 \mathrm{~m})$ of the wavemaker implies there will be some variability in the $y$-direction, although this appears to be quite small in our experiments, see figure 16 in section 4 . Nevertheless, both for the experimental configuration, and with the aim of extrapolating the present results to realistic oceanic situations, it is useful to examine transverse effects in more detail, especially as the theory and numerical simulations described in Grimshaw and Helfrich ${ }^{6,7}$ were only for the one-dimensional configuration. A full account of transverse effects is beyond the scope of this article, and is the subject of an ongoing study. Here, instead we report some numerical simulations of the (rKP) equation derived by Grimshaw ${ }^{4}$,

$$
\left\{A_{t}+\nu A A_{x}+\lambda A_{x x x}\right\}_{x}+\frac{c_{0}}{2}\left(A_{y y}-\frac{f^{2}}{c_{0}^{2}} A\right)=0,
$$

The $A_{y y}$ term takes count of weak transverse linear dispersion, and in its absence this reduces to the Ostrovsky equation (6).

Next note that the transformation

$$
\begin{aligned}
& x=L \tilde{x}, \quad y=K \tilde{y}, \quad t=T \tilde{t}, \quad \eta=M \tilde{\eta} \\
& L^{4}=\frac{\lambda}{\gamma}, \quad K=\frac{c_{0}}{f}, \quad T=\frac{L^{3}}{\lambda}, \quad M=\frac{\lambda}{\nu L^{2}} .
\end{aligned}
$$

takes the rKP equation into itself, but with all coefficients equal to unity. Thus we can replace (16), after omitting the "tilde" symbol, by

$$
\left\{A_{t}+A A_{x}+A_{x x x}\right\}_{x}=A-A_{y y} .
$$

Note that the $y$-scale has been chosen to be equal to the Rossby radius and hence, in a dimensional unscaled form, is much larger than the $x$-extent of the initial pulse. In these transformed coordinates the critical wavenumber is now $k_{m}=(1 / 3)^{1 / 4}=0.760$, and so the length scale of the carrier wave is $2 \pi / k_{m}=8.27$. Also note that the amplitude scales with $\sqrt{\gamma}$, and thus in the original equation (16) the smaller $\gamma$, the more difficult it becomes to find an envelope wave of small amplitude. But that difficulty is avoided here by using the transformed equation (19) instead. However, we need then to note that when using the $\mathrm{KdV} \operatorname{sech}^{2}$-profile as an initial condition, the dependence on $\gamma$ re-emerges in the transformed equation and is now in the initial condition. That is

$$
\begin{gathered}
\eta=a_{0} \operatorname{sech}^{2}(x / D), \quad \nu a_{0} D_{0}^{2}=12 \lambda, \\
\text { becomes } \tilde{\eta}=\tilde{a_{0}} \operatorname{sech}^{2}\left(\tilde{x} / \tilde{D}_{0}\right),
\end{gathered}
$$

$$
\text { where } \quad a_{0}=M \tilde{a_{0}}, D_{0}=L \tilde{D_{0}}, \tilde{a_{0}}{\tilde{D_{0}}}^{2}=12 \text {. }
$$

Thus for a given input amplitude $a_{0}, \tilde{a_{0}}$ varies as $\gamma^{-1 / 2}$ and $\tilde{D}_{0}$ as $\gamma^{1 / 4}$. As $\gamma$ decreases, the input becomes larger and narrower. Equation (19) was integrated numerically using a two-dimensional spectral code with damping regions at large distances upstream, downstream and to each side to absorb radiated waves. The surface was taken to be released from rest with the initial profile

$$
\begin{aligned}
& A(x, y, t=0)=F(x) G(y) \\
& F(x)=a \operatorname{sech}^{2}\left[(a / 12)^{1 / 2} x\right] \\
& G(y)=\frac{1}{2}\left\{\tanh \left[\left(y+y_{e}\right) / y_{w}\right]-\tanh \left[\left(y-y_{e}\right) / y_{w}\right]\right\}
\end{aligned}
$$

This of course is only a qualitative representation of the actual experimental initial conditions. Here we set $y_{e}=20, y_{w}=3$ giving an initial solitary-wave like disturbance that is twenty times as wide in the transverse direction as in the dominant direction of propagation, and thus also giving a Rossby radius of 20 . Figure 4 shows a pseudo-colour plot of the amplitude at a time $t=40$ for the case when the initial amplitude $a=8$. Equation (19) gives the evolution of the disturbance relative to a frame advancing in the positive- $x$ direction with the linear long wave speed. The slowly-evolving weakly-nonlinear disturbance in the $(x, y)$ plane travels more slowly that this long wave speed and thus moves in the negative- $x$ direction. In Figure 4 the disturbance has fallen behind the linear long wave speed by a distance of approximately 150 and decreased in amplitude by a factor of approximately 3 , due to the spreading in the transverse $y$-direction. This dispersive decay can be 


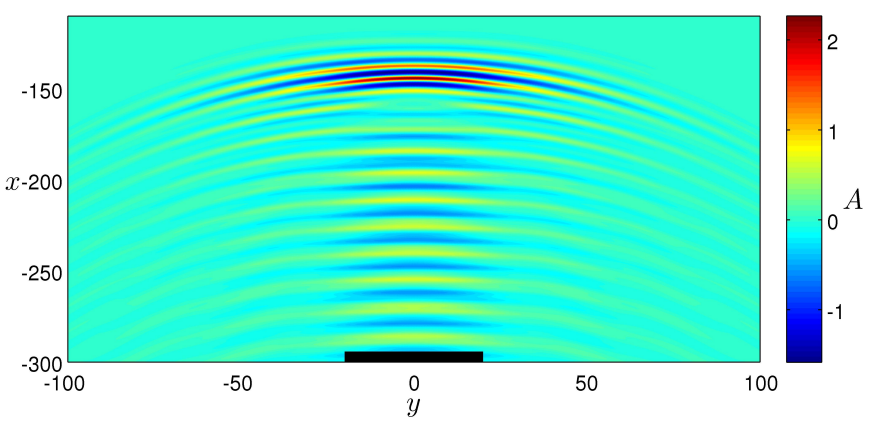

FIG. 4. A pseudo-colour plot of the amplitude from the rKP equation (19) for the initial condition (22) with $a=8, y_{e}=$ $20, y_{w}=3$ at $t=40$. The bar at the base of the figure illustrates the extent of the initial disturbance.

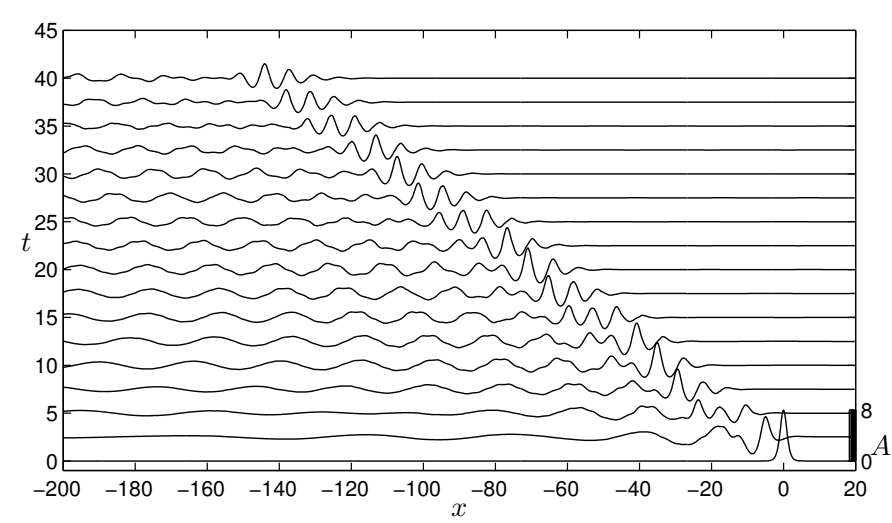

FIG. 5. The amplitude along the centreline $y=0$ from the rKP equation (19) for the initial condition (22) with $a=$ $8, y_{e}=20, y_{w}=3$.

seen in the corresponding time evolution of the amplitude along the centreline in figure 5 . The wave packet has spread in the transverse direction, and collapsed to a smaller amplitude than that found in the analogous Ostrovsky equation simulation, see figure 6 in Grimshaw and Helfrich ${ }^{6}$. Figures 6 and 7 give the corresponding plots for the initial amplitude $a=32$. Figures 6 shows that by $t=30$ the disturbance has collapsed, spread and curved significantly more than in the lower amplitude run. This is also shown in the centreline plot of 7 where the amplitude is seen to have collapsed by a factor of approximately 5 at $t=30$. The most striking feature of these simulations, also found in other analogous simulations, is that although the wave develops considerable curvature, the nonlinear wave packet structure persists along the entire wave profile. The duration of the experiments described below is short compared to the times for the slow evolutions here, and so although packets form rapidly, as shown in figures 5,7 the spreading of figures 4,6 is neither expected nor seen in the experiments, see section III.

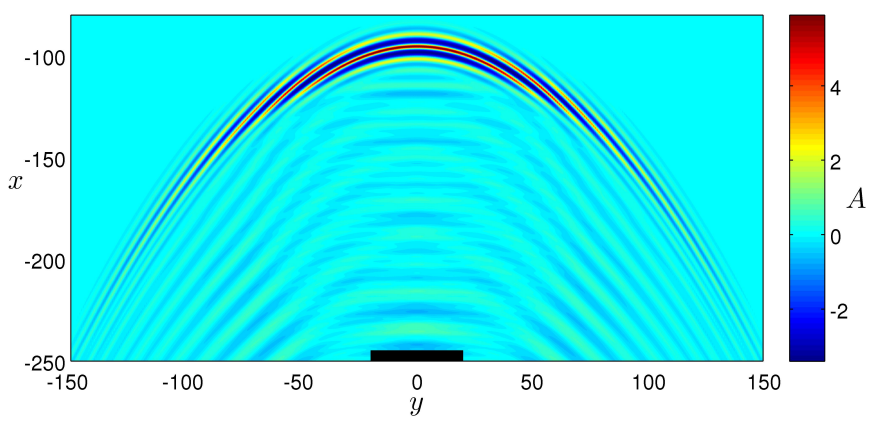

FIG. 6. A pseudo-colour plot of the amplitude from the rKP equation (19) for the initial condition (22) with $a=32, y_{e}=$ $20, y_{w}=3$ at $t=30$. The bar at the base of the figure illustrates the extent of the initial disturbance.

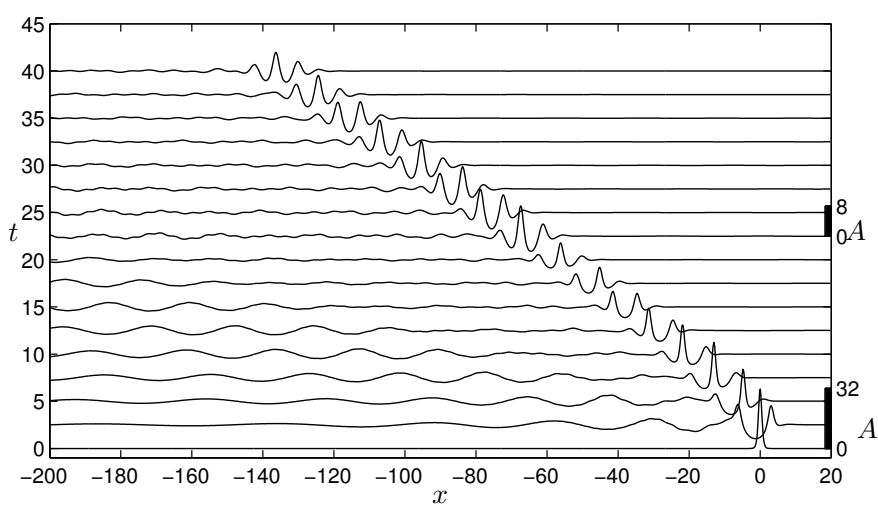

FIG. 7. The amplitude along the centreline $y=0$ from the rKP equation (19) for the initial condition (22) with $a=$ $32, y_{e}=20, y_{w}=3$. The vertical scale for $A$ is doubled for times $t>20$ as shown by the axes at $t=0,22.5$ of height 32,8 respectively.

\section{Numerical simulations of the two-dimensional Navier-Stokes equations}

Next, because the rKP equation (16) is restricted to the weakly nonlinear regime, we also performed some fully nonlinear numerical simulations. The numerical model solves the Boussinesq form of the Navier-Stokes equations using the second-order finite-volume method introduced by Bell and Marcus ${ }^{1}$. However, while the solutions include rotation and the transverse velocity, there is no $y$-dependence, as that study is beyond the scope of this present article.

The simulations were done using conditions that mirror the experiments described in the next section. The background density field is given by

$$
\rho_{0}(z)=\rho_{1}+\frac{\rho_{2}-\rho_{1}}{2}\left(1-\tanh \left[\frac{z-z_{i}}{d_{i}}\right]\right),
$$

where $\rho_{1}$ and $\rho_{2}$ are the densities of the upper and lower layers, respectively, $z_{i}$ is the interface position and $d_{i}$ is 
the interface thickness scale. The simulations are done in a domain with total depth $h=36 \mathrm{~cm}, z_{i}=-6 \mathrm{~cm}, d_{i}=2$ $\mathrm{cm}$, density difference $\Delta \rho=\rho_{2}-\rho_{1}=0.01 \mathrm{gm} \mathrm{cm}^{-3}$. The model is run with no explicit viscosity or density diffusion. The runs are initiated via a lock exchange with a jump in interface depth, $\Delta h_{0}$, and a lock length of 45 $\mathrm{cm}$. The lock is located at $x=0$.

The interface displacement $\eta(x, t)$ determined by the departure of the mid-density surface from its resting position from a case with Coriolis parameter $f=0.14 \mathrm{~s}^{-1}$ and $\Delta h=6 \mathrm{~cm}$ is shown in figure $8 a$. The leading disturbance is a well-defined wave packet with distinct phase and group speeds, followed a less-organized dispersive tail. The frequency-wavenumber spectrum $P(\omega, k)$ (normalized to a maximum of one) computed from the data in figure $13 a$ for $50 \leq x \leq 200$ and $0 \leq t \leq 400$ is plotted in figure $8 b$. The ridge in $P(\omega, k)$ follows the full linear dispersion relation for the background stratification (see (A12) in the Appendix). The location of the maximum group speed, $k_{m}$, from the linear theory is indicated by the circle. A significant fraction of the energy in the wave field is concentrated along the linear dispersion curve for $k \leq k_{m}$. Figure 9 shows time series of $\eta(t)$ at downstream positions that match the experimental measurements (see next section for details). Note that in these two figures quantities are scaled using $h_{1}=6 \mathrm{~cm}$ for lengths and $h_{1} / c_{0}$ for time, where $c_{0}=6.39$ $\mathrm{cm} \mathrm{s} \mathrm{s}^{-1}$ is the linear long wave phase speed found with the background stratification.

\section{EXPERIMENTS}

The combination of asymptotic theory and numerical simulations described by Grimshaw and Helfrich ${ }^{6,7}$ and summarised above provide convincing evidence that an initial KdV solitary wave will decay due to the radiation of inertia-gravity waves, and eventually a new coherent structure emerges, namely a nonlinear wave packet. To examine this hypothesis further, a series of laboratory experiments were conducted in September 2009 using the $13 m$ diameter rotating platform at the LEGI-Coriolis Laboratory in Grenoble. A sketch of the experimental setup is shown in figure 10. All the experiments were conducted with a salt-stratified, two-layer system with the resting upper layer depth $h_{1}=6 \mathrm{~cm}$ and lower layer depth $h_{2}=30 \mathrm{~cm}$. The density difference between the layers was fixed at $0.01 \mathrm{gm} \mathrm{cm}^{-3}$. The measured background stratification is well-approximated by the hyperbolic tangent profile (25) with $z_{i}=-h_{1}$ and the mean interface thickness $d_{i}=2.0 \mathrm{~cm}$. The table rotation periods were

$$
\begin{gathered}
T=4 \pi / f=\infty, 120,90,60,45 s, \\
\text { or } \quad f=0,0.105,0.140,0.209,0.279, s^{-1} .
\end{gathered}
$$

The initial wave was generated by a lock-release from a $5 \mathrm{~m}$ wide by $45 \mathrm{~cm}$ long reservoir that was situated ad-
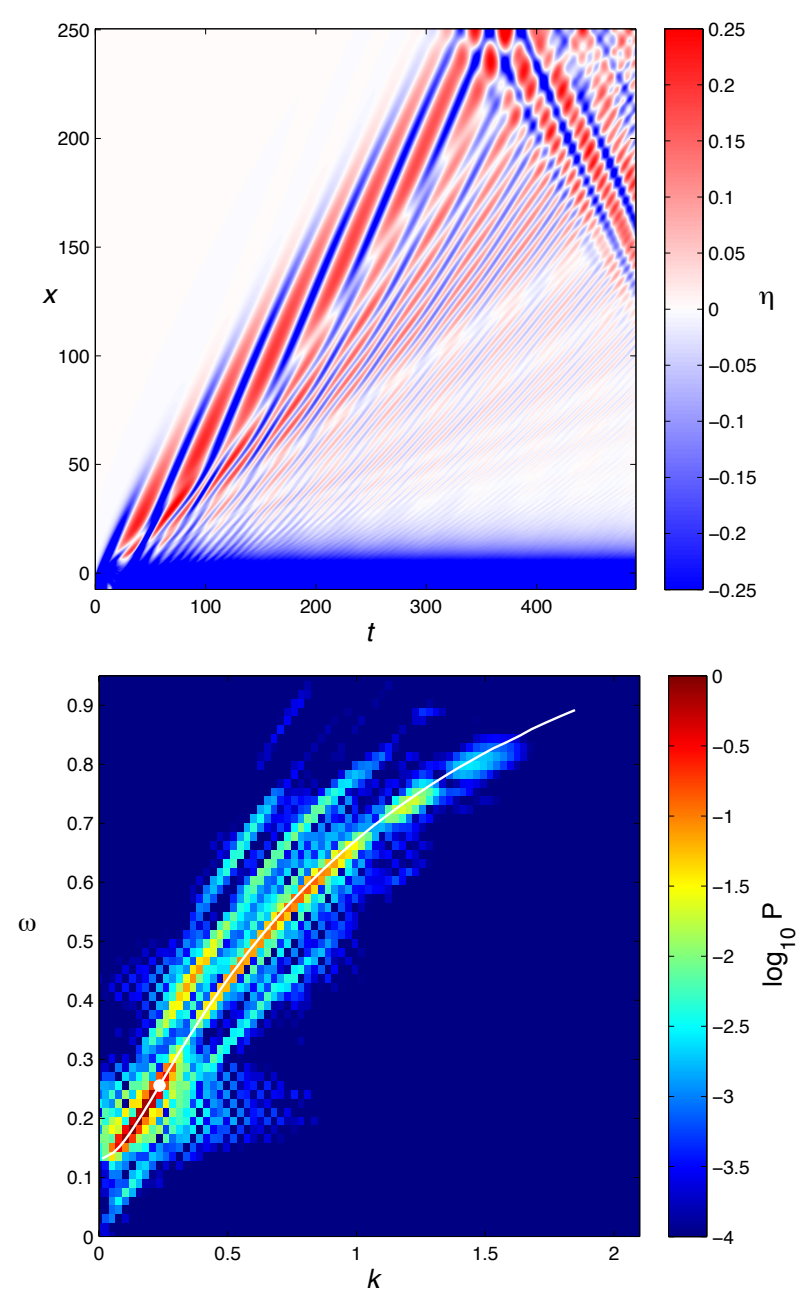

FIG. 8. a) The interfacial displacement $\eta(x, t)$ for the full simulation with $f=0.131$ and $\Delta h=1)$. b) The corresponding $\omega$ - $k$ power spectrum $P$ normalized to a maximum of one. The white curve is the full, linear dispersion relation and the white circle indicates wavenumber of maximum group speed $k_{m}$.

jacent to the outer tank wall. The large width of the reservoir helped to minimize geometric spreading effects. Prior to each experiment the interface within the reservoir was lowered by the addition of fresh water to produce an initial interface elevation difference of approximately $\Delta h_{0}=3,6,9$, or $12 \mathrm{~cm}$. An array of sloping beaches spanning the interface were situated along the far wall of the tank to reduce reflection.

Time series of interface displacement, $\eta(t)$, were obtained using an array of $8-10$ ultrasonic probes spaced along the $x$-axis (origin at the mid-point of the reservoir gate) between $0.75-9.5 \mathrm{~m}$, see figure 10 . The locations were varied between experiments in some cases. These probes use variations in acoustic travel-time between a source and receiver to infer the vertical displacement of the interface. They were calibrated prior to each run 


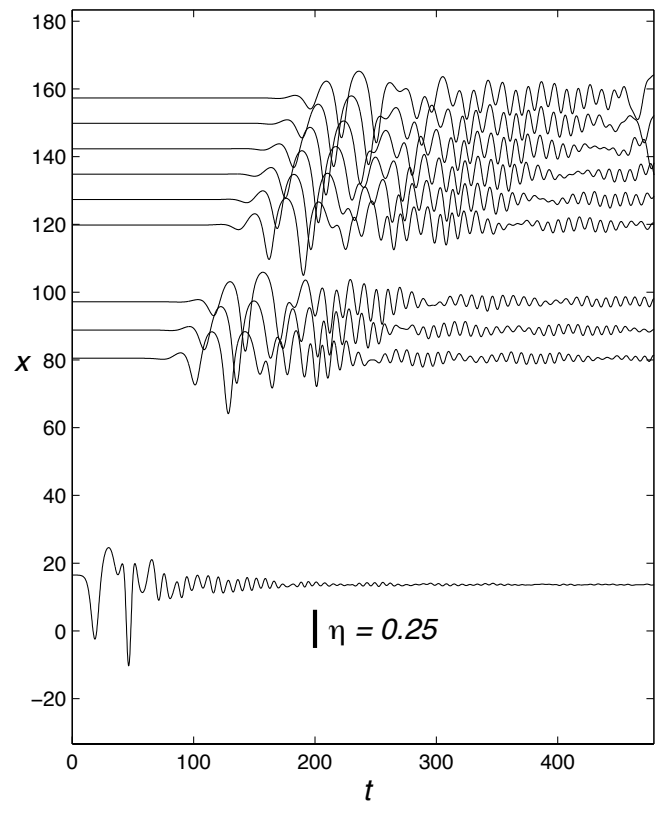

FIG. 9. The interfacial displacement $\eta(t)$ for the simulation in figure 8 . The $x$-location of each probe is indicated by the position of the dashed lines at $t=0$.

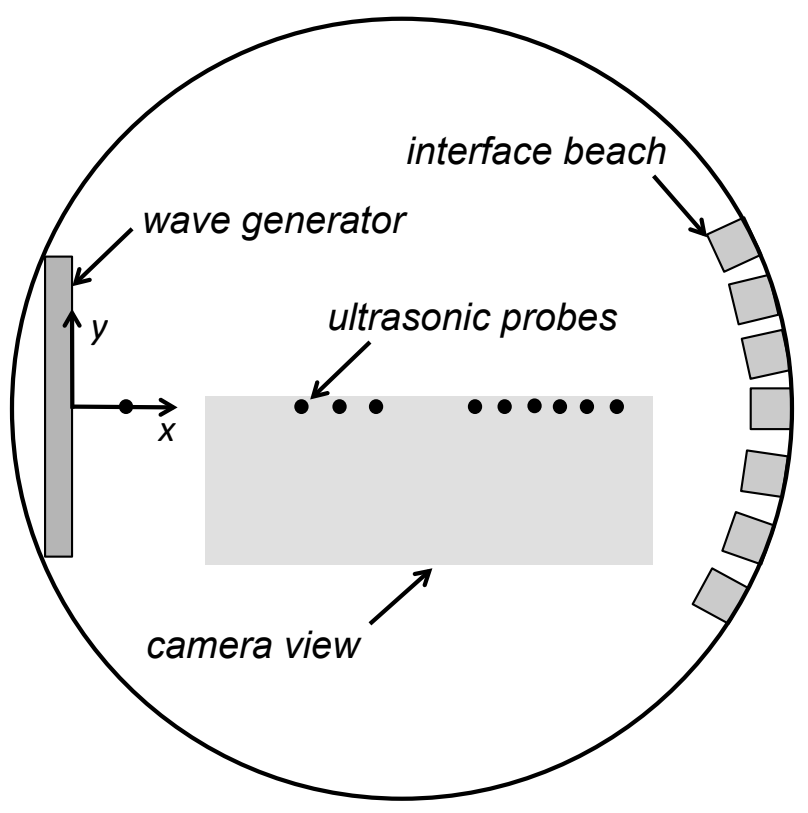

FIG. 10. Plan view sketch of the experimental set-up in the 13 meter diameter Coriolis Platform.

by vertically traversing the probes through the stationary stratification gives the motion equivalent to interface displacements of $\pm 4 \mathrm{~cm}$. The time between calibration and the run was usually just several minutes so that changes in the sound speed profile due to possible temperature change is expected to be very small. Probe output voltages were digitized at $240 \mathrm{~Hz}$ during a run. The

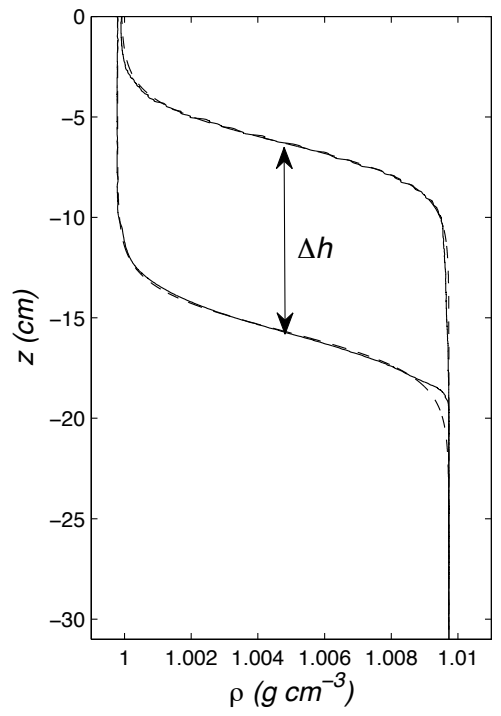

FIG. 11. Example of density profiles just prior to a rotating run inside (lower curve) and outside (upper curve) the reservoir. The dashed lines are best fits of (25). The measured reservoir depth $\Delta h$ is indicated.

conversion between travel time and wave-induced interfacial displacement assumes that the waves simply heave the background stratification up and down and can only capture the first vertical mode. However, because the waves of interest are first-mode and long, this is a good approximation. The wave structure in the transverse $(y)$ direction was obtained by dying the upper layer, lighting from below, and imaging with three overhead CCD cameras at $1 \mathrm{~Hz}$. When the camera images were stitched together after a mapping from the image to spatial coordinates they cover a rectangular area from $y=-2.2-0$ $m$ and $x=2.8-9.7 \mathrm{~m}$. The upper layer depth is proportional to image pixel intensity and was calibrated from a series of images taken as the top layer was added to the tank. The pixel-level estimates of $\eta$ were averaged into $1 \mathrm{~cm}$ by $1 \mathrm{~cm}$ cells. It proved difficult to obtain good calibrations of the image intensity to upper layer depth consistently over the whole imaging area due to the large gradients in the background lighting, variation in ambient lighting, and the filling procedure which only allowed the relationship between image intensity and upper layer depth to be determined for $h_{1} \leq 6 \mathrm{~cm}$. Thus the ultrasonic probes were used to extract quantitative data (wave amplitudes and speeds).

Measurements of the stratification inside and outside the reservoir were made prior to each run using microscale conductivity-temperature probes. Figure 11 shows a typical set of density profiles from a rotating experiment with $\Delta h_{0}=9 \mathrm{~cm}$ and $T=90 \mathrm{~s}$, together with a fit of the hyperbolic tangent function (25). The fitting parameters are the interface depth $z_{i}$ and the in- 
terface thickness scale $\lambda_{i}^{-1}$. The difference in $z_{i}$ between the reservoir and the ambient stratification give the actual reservoir height $\Delta h$. For the non-rotating runs $\lambda_{i}=0.95 \pm 0.06 \mathrm{~cm}^{-1}$. Filling the basin while rotating led to a thicker interface with $\lambda_{i}=0.50 \pm 0.03 \mathrm{~cm}^{-1}$. From these mean values of the stratifications numerical solutions to the Boussinesq version of modal equation (3) gives $c_{0}=6.39$ and $6.65 \mathrm{~cm} \mathrm{~s}^{-1}$ for the rotating and non-rotating runs, respectively. The corresponding coefficients of the KdV equation from (4) are $\nu=-1.18$ $s^{-1}$ and $\lambda=231 \mathrm{~cm}^{3} \mathrm{~s}^{-1}$ with rotation and $\nu=-1.29$ $\mathrm{s}^{-1}$ and $\lambda=220 \mathrm{~cm}^{3} \mathrm{~s}^{-1}$ for the non-rotating cases. For comparison, the two-layer model gives $c_{0}=7 \mathrm{~cm} \mathrm{~s}^{-1}$, $\nu=-1.4 \mathrm{~s}^{-1}$, and $\lambda=210 \mathrm{~cm}^{3} \mathrm{~s}^{-1}$. The differences between the continuous and two-layer models, while relatively small, are important, especially for the rotating runs.

In the presentation of the experimental results that follows, all quantities are nondimensionalized using the nominal upper layer depth $h_{1}=6 \mathrm{~cm}$ and $c_{0}=6.39 \mathrm{~cm}$ $s^{-1}$ from the average continuous stratification. Thus,

$$
\begin{aligned}
(x, y) & =\frac{\left(x^{*}, y^{*}\right)}{h_{1}}, \quad t=\frac{t^{*} c_{0}}{h_{1}}, \quad \eta=\frac{\eta^{*}}{h_{1}}, \quad \Delta h=\frac{\Delta h_{0}}{h_{1}}, \\
c & =\frac{c^{*}}{c_{0}}, \quad k=k^{*} h_{1}, \quad(f, \omega)=\frac{\left(f^{*}, \omega^{*}\right) h_{1}}{c_{0}}
\end{aligned}
$$

where the superscript $*$ indicates the dimensional variable.

Before presenting the experimental results it is important to note that Renouard and Germain ${ }^{15}$ conducted a nearly identical set of experiments. Their reservoir was not as wide and the propagation distance was somewhat shorter. They did not use overhead imaging nor as dense an array of ultrasonic interface probes. Most significantly, they did not have the advantage of the current theoretical model and numerical results to guide their interpretation of the experiments. Rather they attempted to explain their observations as steadily propagating solitary waves, which are known not to exist. However, from their figure 13 it is clear that their experiments produced the same phenomena discussed below. Unfortunately, they did not take or report adequate information about their runs to use here.

Ultrasonic probe recordings of the interface displacement $\eta(t)$ are shown in figure 12 for a non-rotating run with $\Delta h=1.58$. The figure shows the propagation of a solitary wave of depression with nearly steady wave amplitude $\eta_{0}=\min (\eta)=-0.59$ and phase speed $c=1.20$. The amplitude is measured at $x=127.3$ and the phase speed is determined by a least squares fit to the position of the wave crest versus time through the dense array of probes in $x>120$. This speed is quite close to the prediction $c=1.23$ from the KdV model (5). The flat wave crest at $x=157$ is a consequence of data drop-out. The large trailing disturbances for $t>200$ and $x \geq 120$ are primarily reflections from the interface beach. Figure $13 a$ shows an $x-t$ plot of wave amplitude $\eta$ along $y=-3.33$ from the overhead images. Although ampli-

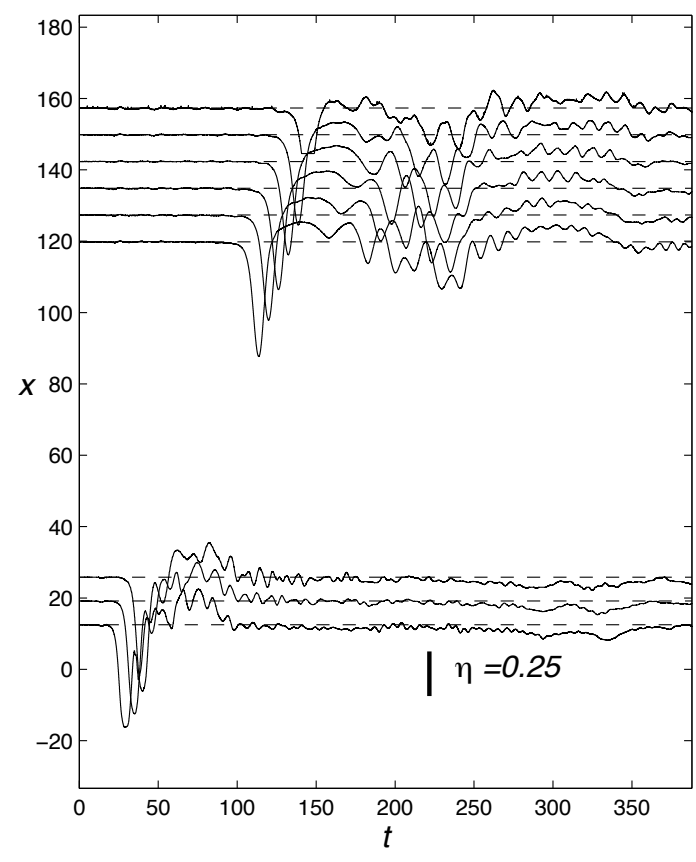

FIG. 12. The interfacial displacement $\eta(t)$ from the ultrasonic probes for a non-rotating experiment with $\Delta h=1.58$. The $x$-location of each probe is indicated by the position of the dashed lines at $t=0$.

tudes from the camera images are less reliable than the interface probes, the figure clearly shows that the leading solitary wave propagates at a nearly constant speed. The frequency-wavenumber spectrum $P(\omega, k)$ (normalized to a maximum of one) computed from the data in figure $13 a$ is plotted in figure $13 b$. The solitary wave shows up as the linear ridge of high spectral amplitudes. The dispersive part of the spectrum agrees well with the full, linear dispersion relation (see (A12) in the Appendix) for the average non-rotating stratification. The non-rotating runs with $\Delta h=1.08$ and 2.13 confirm that the generation mechanism produces a single, steadily propagating solitary wave of depression and a dispersive tail.

The effect of rotation is illustrated in figure III for an experiment with $\Delta h=1.57$ and $f=0.131(T=90 \mathrm{~s})$. The wave recorded at the first probe, $x=16.7$, shows a leading solitary-like depression of the interface followed by a substantial dispersive tail. Once the disturbance reaches the probe at $x=88$ a leading wave packet, rather than a solitary wave, has emerged. The propagation of the packet through the downstream probe array shows clearly an identifiable carrier wave with a phase speed that is faster than the group speed of the packet. This is in qualitative agreement with the theoretical discussion in section 2 .

The phase speed of the carrier wave in figure III is $c=1.10$ and was found by fitting lines to the $x-t$ positions of individual wave crests and troughs for $x>120$. The slopes of these fits are then averaged to give $c$. The 

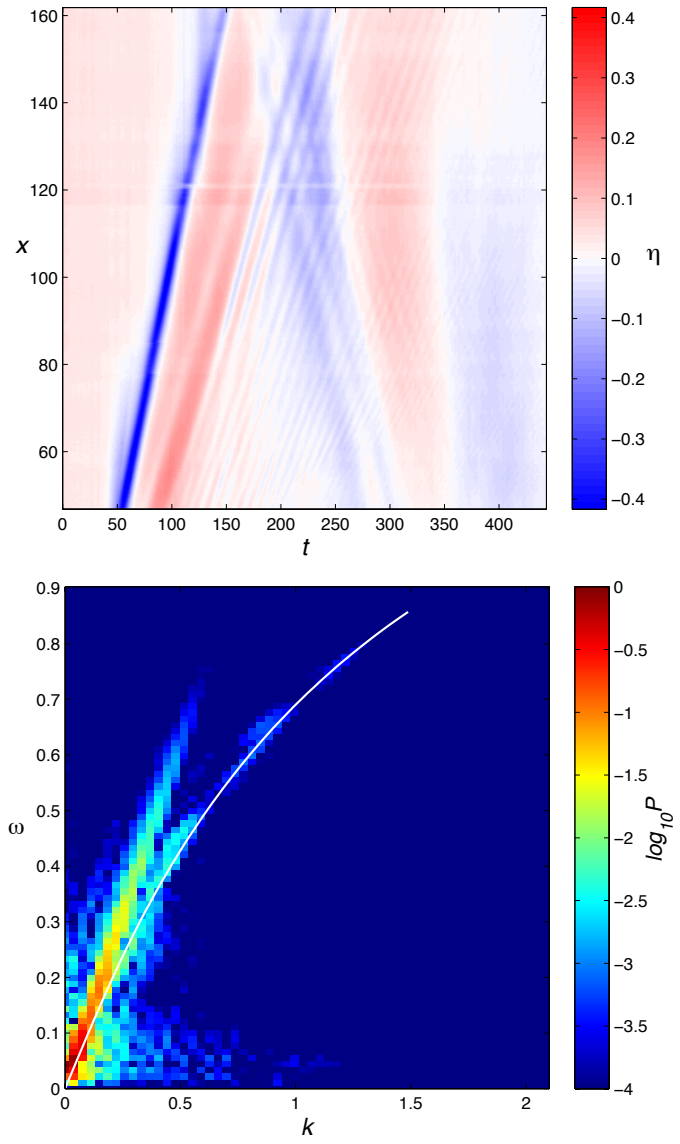

FIG. 13. a) The interfacial displacement $\eta(x, t)$ along $y=$ -3.33 from the overhead cameras for the experiment in figure $12(f=0$ and $\Delta h=1.58)$. b) The corresponding $\omega$ - $k$ power spectrum $P$ normalized to a maximum of one. The white curve is the full, linear dispersion relation.

uncertainty of the speed determined with this method is $\pm 5 \%$ based on the standard deviation of the phase speed estimates for individual crests and troughs and the quality of the linear fits. The carrier wave wavelength $\Lambda=2 \pi / k=c t_{c}=27.4$ is found from the average wave period $t_{c}$ in the packet. The packet group speed $c_{g}=0.70$ is found by fitting a cubic spline wave crests (or troughs) at a fixed $x \geq 120$. The maximum of this spline function gives the $x$ - $t$ locations of the packet which are fitted to a line. The average from the trough and crest packet locations gives $c_{g}$. The uncertainty in the group speed is estimated to be $\pm 10 \%$. The group speed was also found by fitting a line through the $x$ - $t$ locations of the leading edge of the packet. This method is more subjective than the spline fit, but gives comparable values of $c_{g}$.

Figure $15 a$ shows an $x$ - $t$ plot of $\eta$ along $y=-3.33$ from the overhead images. Again, a well-defined localized, leading packet with different phase and group speeds is clear. This is again consistent with the probe data, but provides a more complete view of the leading packet structure and the distinct phase and group speeds. It

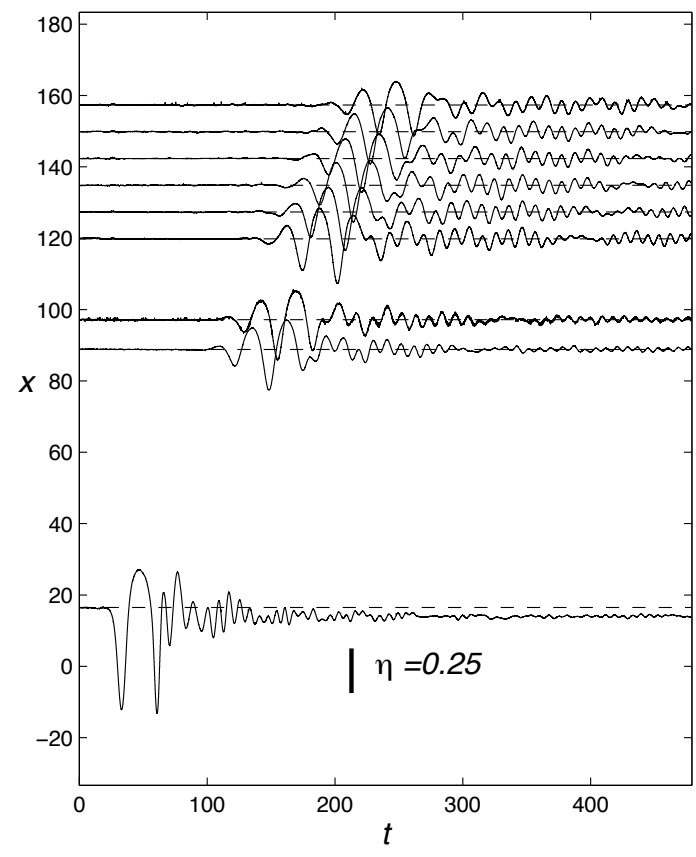

FIG. 14. The interfacial displacement $\eta(t)$ from the ultrasonic probes for a rotating experiment with $f=0.131$ and $\Delta h=$ 1.57 . The $x$-location of each probe is indicated by the position of the dashed lines at $t=0$.

also shows clearly that the packet is followed by smaller amplitude waves with different wave numbers. The spatial modulation of the amplitude of any individual trough or crest within the packet is a consequence of the problematic calibration of the imaging. The systematic variations in interface displacement, for example the v-shaped features along $x=125$ and the horizontal striping, are caused by ambient lighting variations introduced by the platform rotation. The corresponding spectrum $P(\omega, k)$ is plotted in figure $15 b$. The ridge in $P(\omega, k)$ follows the full linear dispersion relation computed with the average, continuous stratification for the rotating runs. The location of the maximum linear group speed, $k_{m}$, from the linear theory is indicated by the circle. In contrast to the non-rotating example in figure $13 b$, most of the energy in the wave field is concentrated along the linear dispersion curve for $k \leq k_{m}$.

The experimental results in figures and 15 compare quite well to the numerical simulation with the same stratification and rotation rate shown in figure 8 and 9 . However, the simulation was done with $\Delta h=1$. The need for a reduced $\Delta h$ to obtain comparable wave amplitudes is potentially a consequence of geometric spreading absent in the calculations. It is also due to the inability of the numerical model to accurately simulate the threedimensional turbulent mixing and dissipation produced by the sudden lock release.

The overhead imaging can also be used to examine the horizontal structure of the interfacial displacement. 

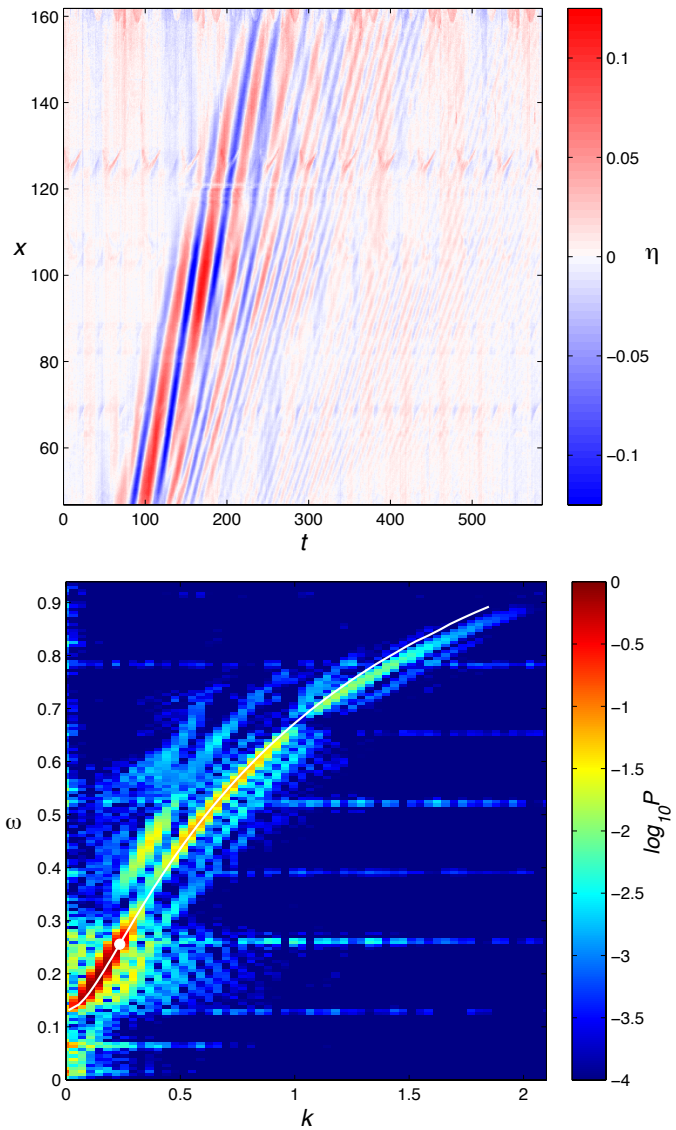

FIG. 15. a) The interfacial displacement $\eta(x, t)$ along $y=$ -3.33 from the overhead cameras for the rotating experiment in figure III ( $f=0.131$ and $\Delta h=1.57)$. b) The corresponding $\omega-k$ power spectrum $P$ normalized to a maximum of one. The white curve is the full, linear dispersion relation. The white circle indicates wavenumber of maximum group speed $k_{m}$.

Figure 16 shows $\eta(x, y)$ at $t=181$ for the experiment in figure III. Geometric spreading due to the finite extent $(|y| \leq 41.7)$ of the reservoir is apparent. However, the transverse variations of $\eta$ in the centre of the domain, $|y|<10$, are relatively small. Thus to leading order the effects of geometric spreading can be ignored. Waves properties measured along the $x$ axis with the ultrasonic probes are assumed independent of $y$, as in the theory and numerical modeling.

The interfacial responses for all the runs are summarized in figures 17-20 for $\Delta h_{0}=0.5,0.1,0.15$, and 2 respectively. Each figure shows $\eta(t)$ from the ultrasonic probes at $x=16.7$ and $x=127.3$ for the same $\Delta h_{0}$ across the range of rotation periods $T=4 \pi / f$ explored. The time origin of each run has been shifted so that the first local minimum of $\eta$ at $x=16.7$ occurs at $t=0$. The figures show that the character of the response is largely independent of $\Delta h_{0}$. The non-rotating experiments produce a single solitary wave that propagates

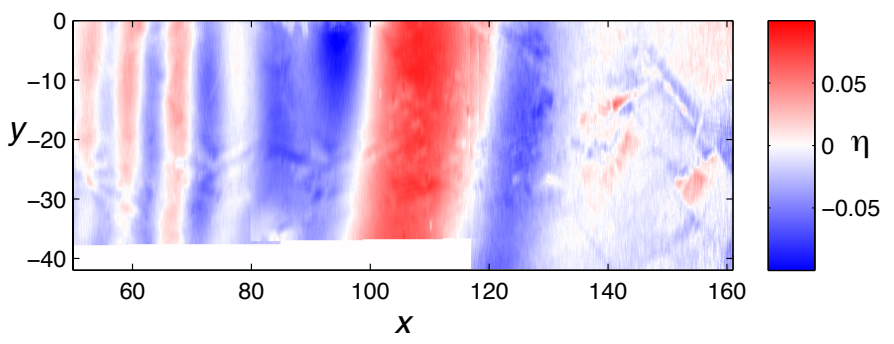

FIG. 16. Plan view of the interfacial displacement $\eta(x, y)$ at $t=181$ from the experiment in figure III $(f=0.131$ and $\Delta h=1.57)$. The waves are propagating from left to right.
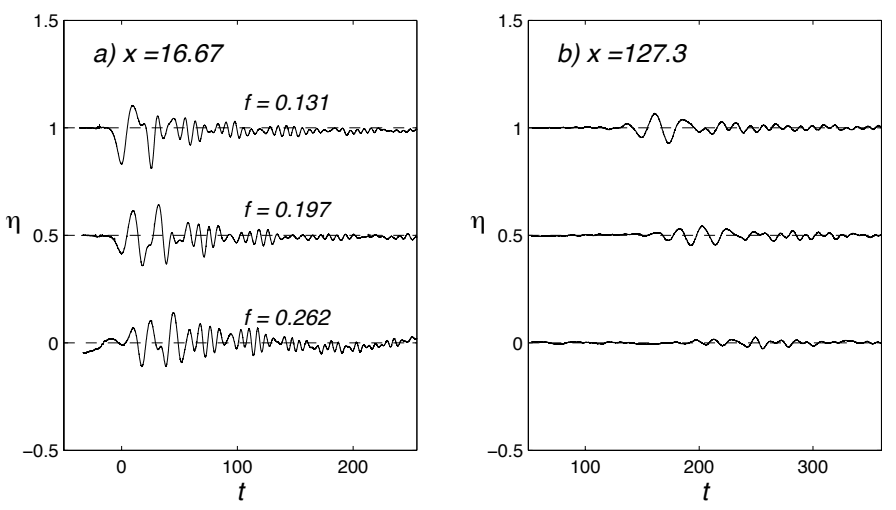

FIG. 17. Interfacial displacement $\eta(t)$ at a) $x=16.7$ and b) $x=127.3$ from experiments with $\Delta h_{0}=0.5$ and $f$ as indicated. The time in each run has been shifted so that the first local minimum in $\eta$ at $x=1.67$ occurs at $t=0$. Each run is offset by one in $\eta$.
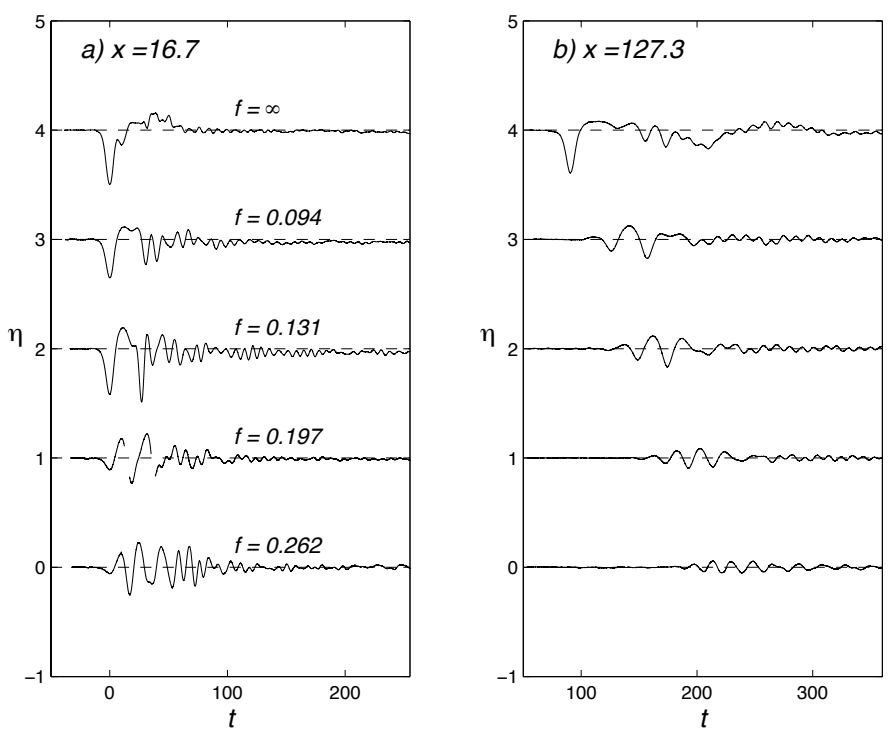

FIG. 18. Same as figure 17 except $\Delta h_{0}=1$. 

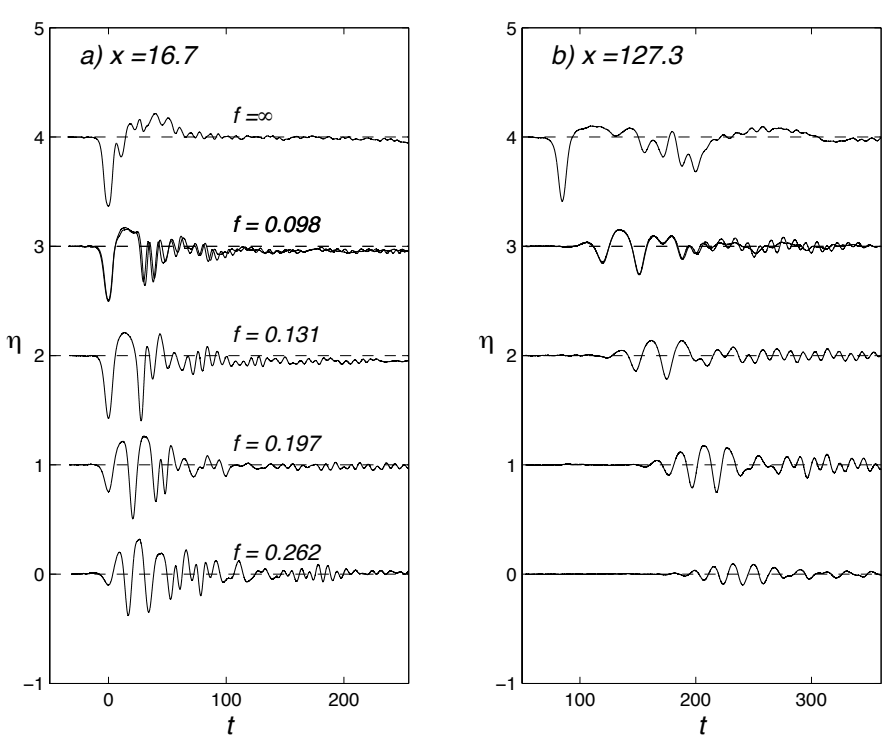

FIG. 19. Same as figure 17 except $\Delta h_{0}=1.5$.
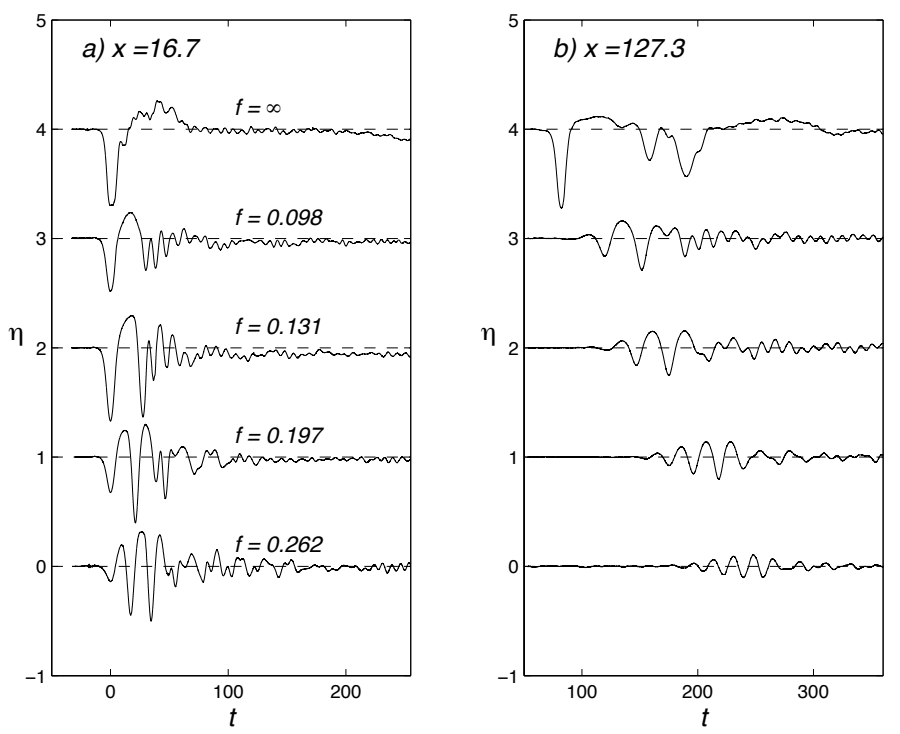

FIG. 20. Same as figure 17 except $\Delta h_{0}=2$.

with little change in amplitude. The introduction of rotation leads to the formation of a well-defined wave packet by $x=127.3$, and for $f>0.19$ the packet structure is evident by the first probe at $x=16.7$. The leading packet group speeds decrease with $f$, while the carrier wave frequency increases. Also, two runs were made at $\Delta h_{0}=1.5$ and $f=0.098$. Both are plotted in figure 19. Except for the trailing, high frequency part of the signals, these two runs are nearly identical indicating the the experiments are repeatable.

The measured carrier wave length $\Lambda$, phase speed $c$ and packet group speed $c_{g}$ are shown as functions of $f$
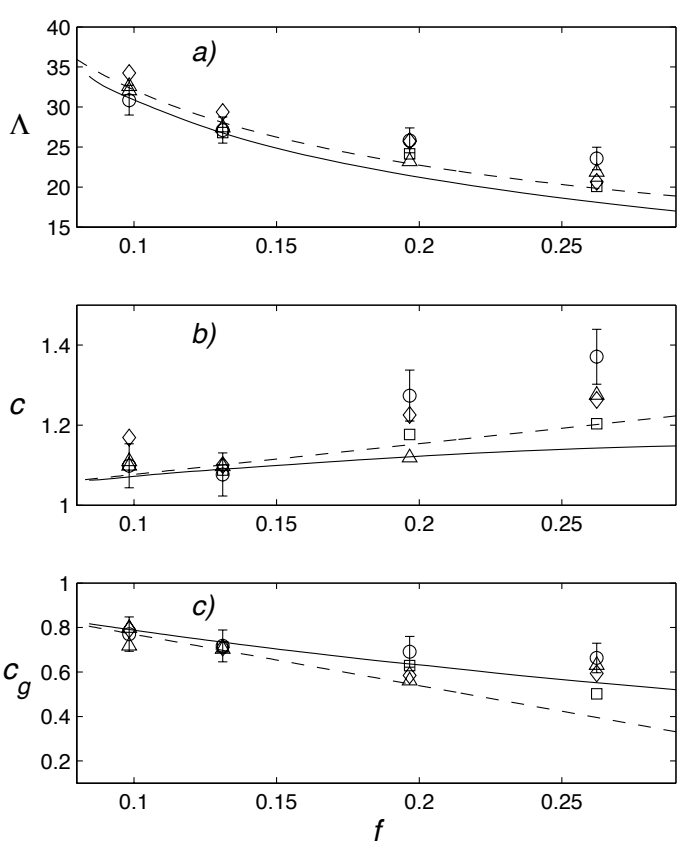

FIG. 21. Experimental results for a) the carrier wave length $\Lambda=2 \pi / k$, b) phase speed $c$, and c) packet group speed $c_{g}$ versus $f$. The symbols indicate $\Delta h_{0}=0.5$ (square), 1 (circle), 1.5 (triangle), and 2 (diamond). Representative error bars are shown for $\Delta h_{0}=1$. Theoretical predictions for $\Lambda_{m}, c_{m}$, and $c_{g m}$ using average stratification are shown for the Ostrovsky equation (dashed) and the full dispersion relation (solid).

for all the rotating experiments in figure 21. The figure also shows the predictions for the carrier wave length, $2 \pi / k_{m}$, phase speed, $c_{p m}$, and the maximum group speed $c_{g m}$ from the Ostrovsky equation dispersion relation (9). The same quantities from the full, linear dispersion relation from solutions of (A12) are also plotted. Both sets of theoretical curves use the average, continuous stratification from (25) for the rotating runs. Overall, the agreement between the theoretical predictions and the experimental data is very good. The carrier waves tend to be slightly longer than predicted, especially as $f$ increases and consistent with the spectrum in figure $15 b$. The phase speeds are generally faster than predicted, while the group speeds agree well with the theory, especially the full linear dispersion relation.

The results in figure 21 do not indicate any systematic dependence on $\Delta h_{0}$, and hence wave amplitude. This is shown more explicitly in figure 22 where the phase and group speeds are plotted as functions of the wave amplitude $\eta_{0}=\min (\eta)$ at $x=127.3$. First, the phase speeds of the solitary waves in the non-rotating runs are plotted in figure $22 a$ along with the prediction from the KdV theory (5) using the average, non-rotating stratification. The agreement with the KdV model is quite good. In contrast, the rotating results for $c$ and $c_{g}$ in figures $22 b$ and $c$, respectively, do not show any clear dependency on $\eta_{0}$ for a fixed $f$. This could be a consequence of the ex- 

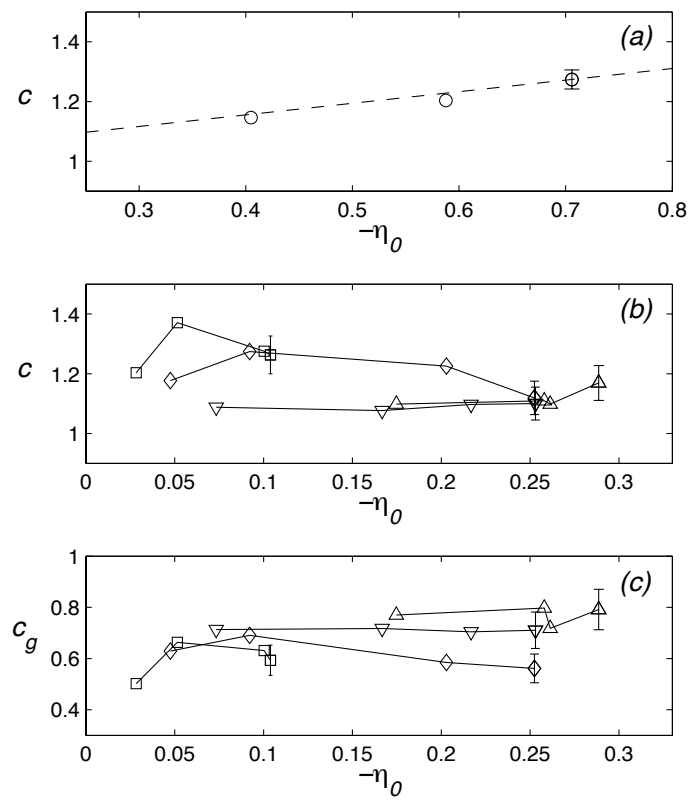

FIG. 22. a) Measured phase speed $c$ versus wave amplitude $\eta_{0}=\min (\eta)$ for the non-rotating runs. The dashed line is the prediction from the KdV model (5). b) and c) show the measured phase, $c$, and group, $c_{g}$, speeds versus amplitude $\eta_{0}$ for the rotating runs. The symbols in (b) and (c) indicate $f=$ 0.098 (triangle up), 0.131 (triangle down), 0.196 (diamond), and 0.262 (square).

perimental errors and uncertainty. It is more difficult to measure carrier wave phase and packet group speeds than the phase speed for a solitary wave. However, numerical solutions of the Ostrovsky equation (6) by Grimshaw and Helfrich $^{6}$ showed that the the group speed was relatively insensitive to the packet amplitude (see their Figure 9). In figure 23 we show comparisons of the packet speed $V$ and wavenumber $K$ from the theoretical result (15), from the numerical solutions of (6) by Grimshaw and Helfrich ${ }^{6}$, and from the experiments. The experimental wavenumber was determined by fitting a $\operatorname{sech}\left(K c_{g} t\right)$ envelope to the leading packet at $x=127.3$. While there is clearly some discrepancy between the theoretical values and the numerical and experimental values, the latter two are in quite good agreement.

As shown in figure 21, the measured values of $\Lambda, c$, and $c_{g}$ of the leading packets are all close to those predicted from the linear dispersion relation for a wave with the maximum group speed. While this is consistent with the nonlinear theory, it is also consistent with linear theory and by itself is not a distinguishing feature of a nonlinear response. However, the distinct structure of the leading packet in figures 17-20 with maximum wave amplitude behind the leading edge and fore-aft symmetry of the packet envelope, evident especially as $f$ increases, is indicative of nonlinear behaviour. Linear analysis predicts the largest amplitude at the leading edge with oscillations decaying monotonically behind, described by
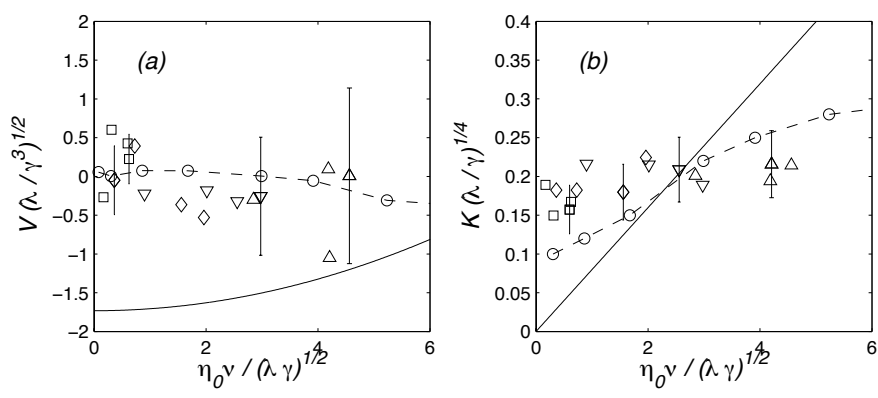

FIG. 23. The nonlinear correction to the group speed $V$ versus packet amplitude $\eta_{0}$. b) The packet wavenumber $K$ versus $\eta_{0}$. The results are non-dimensionalized using the scaling (17). The solid lines show the predictions from the NLS model. The circles with the connecting dashed lines show results from the numerical solutions of the Ostrovsky equation (6). The experimental results are indicated as $f=0.098$ (triangle up), 0.131 (triangledown), 0.196 (diamond), and 0.262 (square).

an Airy function, (see $\S 2.3$ in Grimshaw and Helfrich ${ }^{8}$, whereas the symmetric envelope is in qualitative agreement with the nonlinear theoretical and numerical results above. This together with the agreement between the experiments and numerical solutions in figure $23 \mathrm{im}-$ plies that packets are a consequence of nonlinearity and qualitatively described by the extended NLS model and numerical solutions of the Ostrovsky equation.

\section{DISCUSSION}

Our recent numerical modeling and theoretical studies $^{6,7,9}$ have shown that the long-time outcome of weak rotational influence on an initial solitary wave is the development of a leading nonlinear wave packet described by an extended nonlinear Schrodinger equation. The packet carrier wave characteristics are determined by the maximum group velocity of the linear dispersion relation. These theoretical results have been tested through a series of laboratory experiments on the rotating 13 meter Coriolis Platform in Grenoble. The experiments were conducted by a lock-exchange mechanism that for no rotation produced single solitary waves that propagate steadily and in good agreement with the KdV solitary wave amplitude dispersion relation. The introduction of rotation in all cases led to the formation of a leading nonlinear wave packet with carrier wavenumber, carrier phase speed, and packet group speed in good agreement with the theoretical predictions from the linear dispersion relation calculated using the continuous stratification of the experiments. Inclusion of the effects of finite wavelength in the dispersion relation generally improved the agreement. The nonlinearity of the leading packet is demonstrated by their fore-aft symmetry, whereas a linear theory would lead to an Airy function structure. The nonlinear modification to the packet group speed 
and the packet envelop wavenumber did not agree well with the extended NLS model, but did agree with those from previous numerical solutions to the Ostrovsky equation, implying that the extended NLS model captures the qualitative behavior, but not the quantitative details of the nonlinear development. See Grimshaw andHelfrich ${ }^{6}$ for a discussion of the limitations on the NLS model.

For low rotation rates the initial wave signal close to the lock was a leading solitary wave followed by radiating inertia-gravity waves. This is consistent with the idealized theory and modelling which were initialised with a $\mathrm{KdV}$ solitary wave. Increasing the rotation rate in the experiments reduced the leading solitary wave to the point where it was no longer identifiable in the interface displacement recored at the probe closest to the lock, yet the nonlinear packets still emerged. Thus the development of the packet does not require an initial solitary wave, but is the expected outcome of a general initial condition, in this case a geostrophic adjustment, that radiates inertia-gravity waves followed by the development of leading wave packet. This may be important in applications to the ocean since it demonstrates that the packets need not evolve exclusively from the radiative decay of an internal solitary wave. However, we note that Euler equation simulations by Stastna et al ${ }^{16}$ show that the timescale for this development can be unrealistically long in certain oceanic conditions. In general, initial conditions are expected to lead to wave packets on a timescale set by the requirement that the inertiagravity wave with the maximum group speed can emerge from the initial disturbance. This may be less than the time for the packets to form from a solitary wave since it does not involve the initial radiative decay phase.

We are not aware of clear oceanic observations of these nonlinear wave packets. However, there are some indications of this behavior. In particular, Vlasenko et al ${ }^{17}$ call attention to observations by Garrido et $\mathrm{al}^{3}$ of solitary-like wave packets propagating from the Strait of Gibraltar into the Alboran Sea that do not have the classic rankordered structure typical of non-rotating KdV dynamics. Rather, in some cases the packets have an envelope structure more in character with the nonlinear wave packets found in our experiments. Vlasenko et al ${ }^{17}$ used a threedimensional non-hydrostatic numerical model to study wave packets and concluded that the non-rank-ordered packets are a consequence of wave scattering and reflection by the complex topography of the Strait. However, they do find that rotation is required for these packets to form in their model simulations. The apparent carrier wavelength $\Lambda \approx 3-4 \mathrm{~km}$ in figure 14 of Vlasenko et $\mathrm{al}^{17}$. The carrier wavelength $\Lambda=2 \pi / k_{m}=2 \pi(3 \lambda / \gamma)^{1 / 4} \approx 7$ $\mathrm{km}$ from (9) using the two-layer $\mathrm{KdV}$ coefficients from (4) with $h_{1}=60 \mathrm{~m}, h_{2}=240 \mathrm{~m}, g^{\prime}=0.02 \mathrm{~m} \mathrm{~s}^{-2}$, and $f=8.510^{-5} s^{-1}$ representative of their calculations. So while there is a factor of two difference between the theory and this one example, the results of Vlasenko et $\mathrm{al}^{17}$ are nevertheless suggestive of the dynamics demonstrated by our experiments, especially in light of the rapid, robust wave packet formation from general initial conditions (e.g., topographically scattered internal solitary waves in the Strait).

\section{Acknowledgment}

This work has been supported by European Community's Sixth Framework Programme through the grant to the budget of the Integrated Infrastructure Initiative HYDRALAB III within the Transnational Access Activities, Contract no. 022441. KRH was supported by grant N00014-09-1-0227 from the Office of Naval Research. We wish to thank the excellent team at the LEGI Coriolis Platform, Joel Sommeria, Louis Gostiaux, Henri Didelle, and Samuel Viboud, for their help in setting up and conducting the experiments.

\section{Appendix A: Full linear dispersion relation for two-layer and continuously stratified fluids}

For a two-layer fluid, the full linear dispersion relation in the absence of rotation and in the Boussinesq approximation is

$$
c^{2}=\frac{\omega^{2}}{k^{2}}=\frac{g^{\prime}}{k\left[\operatorname{coth} k h_{1}+k \operatorname{coth} k h_{2}\right]} .
$$

Here $h_{1,2}$ are the upper and lower layer depths. In the long wave limit, $k \rightarrow 0$, and for right-going waves, these reduce to

$$
c \approx c_{0}\left(1-\frac{k^{2} h_{1} h_{2}}{6}\right), \quad c_{0}^{2}=\frac{g^{\prime} h_{1} h_{2}}{h_{1}+h_{2}} .
$$

Thus, for the $\mathrm{KdV}$ equation (1) the coefficient $\lambda=$ $c_{0} h_{1} h_{2} / 6$. In the above equations and throughout the rest of the Appendix dimensional variables are used.

When rotation is included, the governing equations for a linearized two-layer configuration are in each layer are

$$
\begin{aligned}
u_{j t t}+f^{2} u_{j}+q_{j x t} & =0, \\
w_{j t}+q_{j z} & =0, \\
u_{j x}+w_{j z} & =0,
\end{aligned}
$$

where $j=1,2$ in the upper layer $-h_{1}<z<0$ and lower layer $-h_{2}-h_{1}<z<-h_{1}$ respectively. At the upper rigid boundary $w_{1}=0, z=0$ and at the lower rigid boundary $w_{2}=0, z=-h_{2}-h_{1}$. Here in the upper layer the full pressure is $-\rho_{1} g\left(z+h_{1}\right)+\rho_{1} q_{1}$ and in the lower layer the full pressure is $-\rho_{2} g\left(z+h_{1}\right)+\rho_{2} q_{2}$. At the interface, using the Boussinesq approximation $\rho_{2}-\rho_{1}<<\rho_{2}$,

$$
\zeta_{t}=w_{1}=w_{2}, \quad \rho_{2} q_{2}-\rho_{1} q_{1}=g\left(\rho_{2}-\rho_{1}\right) \zeta .
$$

Then, seeking solutions proportional to $\exp (i k x-i \omega t)$ we get that

$$
w_{j z z}=\kappa^{2} w_{j}, \quad \kappa^{2}=\frac{\omega^{2} k^{2}}{\omega^{2}-f^{2}}
$$


while $q_{j}$ satisfies the same equation. Hence

$w_{1}=i \omega a \frac{\sinh (\kappa z)}{\sinh \left(\kappa h_{1}\right)}, \quad w_{2}=-i \omega a \frac{\sinh \left(\kappa\left(z+h_{1}+h_{2}\right)\right)}{\sinh \left(\kappa h_{2}\right)}$,

where the kinematic boundary condition, and the upper and lower rigid lid boundary conditions have been imposed. Note that here $a$ is the interface amplitude, and that $q_{j}=i \omega w_{j z} / \kappa^{2}$. Finally, imposing the dynamic boundary condition we get the dispersion relation

$$
\omega^{2}=\frac{g^{\prime} \kappa}{\operatorname{coth}\left(\kappa h_{2}\right)+r \operatorname{coth}\left(\kappa h_{1}\right)},
$$

where $r=\rho_{1} / \rho_{2}, g^{\prime}=g\left(\rho_{2}-\rho_{1}\right) / \rho_{2}$. The known result for water waves follows by putting $\rho_{1}=0$, while the Boussinseq approximation sets $r=1$, so that

$\omega^{2}=\frac{g^{\prime} \kappa}{\operatorname{coth}\left(\kappa h_{2}\right)+\operatorname{coth}\left(\kappa h_{1}\right)}=\frac{g^{\prime} \kappa \sinh \left(\kappa h_{1}\right) \sinh \left(\kappa h_{2}\right)}{\sinh \left(\kappa\left(h_{1}+h_{2}\right)\right)}$.

In the absence of rotation $\kappa=k$, and then (A10) collapses to (A1). We shall assume we are only concerned with solutions for which $\omega^{2}>f^{2}$, so that $\kappa$ is real-valued. In the long-wave limit $k \rightarrow 0$, we may write $\omega^{2} \approx f^{2}+c_{0}^{2} k^{2}+$ $O\left(k^{4}\right)$, so that $\kappa \approx f / c$, and then (A10) collapses to

$$
f c_{0} \approx \frac{g^{\prime}}{\operatorname{coth}\left(f h_{2} / c_{0}\right)+\operatorname{coth}\left(f h_{1} / c_{0}\right)},
$$

which is an implicit expression for $c_{0}$. However, making the further approximation that $f h_{1,2}<<c_{0}$, we recover the long-wave limit of the non-rotating dispersion relation (A2). However, the experimental parameters where $f=4 \pi / T \mathrm{sec}^{-1}, h_{1}=6 \mathrm{~cm}, h_{2}=30 \mathrm{~cm}, c_{0}=7 \mathrm{~cm} \mathrm{sec}^{-1}$ suggest that this last approximation may not be valid here. A correction when $f h_{1,2}<<c_{0}$ yields instead

$$
c_{0}^{2} \approx \frac{g^{\prime} h_{1} h_{2}}{\left(h_{1}+h_{2}\right)\left(1+f^{2}\left(h_{1}+h_{2}\right) / 3 g^{\prime}\right)},
$$

showing that the effect of $f$ is to reduce the phase speed. For the experimental parameters the rotational correction is $0.04,0.29$ when $T=120,45 \mathrm{sec}$ respectively. In general, equation (A11) has a unique solution for $c_{0}$ which decreases as $f^{2}$ increases, when $h_{1,2}$ are fixed.

When the fluid is continuously stratified the full linear dispersion relation $\omega(k)$ with rotation must be found from the modal problem, see Pedlosky ${ }^{14}$

$$
\begin{aligned}
& \frac{d^{2} \phi}{d z^{2}}+k^{2}\left(\frac{N^{2}(z)^{2}-\omega^{2}}{\omega^{2}-f^{2}}\right) \phi=0, \\
& \text { for }-h<z<0, \text { and } \phi(-h)=\phi(0)=0 .
\end{aligned}
$$

Here we have used the Boussinesq approximation where $N^{2}=-\left(g / \rho_{1}\right) d \rho_{0} / d z$ where $\rho_{1}$ is a reference density. The linear dispersion relation $\omega=\omega(k)$ for the first vertical mode, when $f$ and $\rho_{0}(z)$ are specified, is found from a numerical solution of (A12).

${ }^{1}$ Bell, J. B. and Marcus, D. L. (1992). A second-order projection method for variable-density flows. J. Comp. Phys., 101:334-348. ${ }^{2}$ Galkin, V. N. and Stepanyants, Y. A. (1991). On the existence of stationary solitary waves in a rotating fluid. J. Appl. Maths. Mech., 55:939-943.

${ }^{3}$ Garrido, J. C. S., Lafuente, J. G., Aldeanueva, F. C., Baquerizo, A., and Sanino, G. (2008). Time-spatial variability observed in velocity of propagation of the internal bore in the strait of gibraltar. J. Geophys. Res., 113:C07034.

${ }^{4}$ Grimshaw, R. (1985). Evolution equations for weakly nonlinear, long internal waves in a rotating fluid. Stud. Appl. Math., 73:133.

${ }^{5}$ Grimshaw, R. (2001). Internal solitary waves. In Grimshaw, R., editor, Environmental Stratified Flows, pages 1-27. Kluwer.

${ }^{6}$ Grimshaw, R. and Helfrich, K. R. (2008). Long-time solutions of the ostrovsky equation. Stud. Appl. Math., 121:71-88.

${ }^{7}$ Grimshaw, R. and Helfrich, K. R. (2012). The effect of rotation on internal solitary waves. IMA J. Appl. Math., 77:326-339.

${ }^{8}$ Grimshaw, R. H. J., Ostrovsky, L. A., Shrira, V. I., and Stepanyants, Y. A. (1998). Long nonlinear surface and internal gravity waves in a rotating ocean. Surveys in Geophysics, 19:289-338.

${ }^{9}$ Helfrich, K. R. (2007). Decay and return of internal solitary waves with rotation. Phys. Fluids., 19:026601.

${ }^{10}$ Helfrich, K. R. and Melville, W. K. (2006). Long nonlinear internal waves. Ann. Rev. Fluid Mech., 38:395-425.

${ }^{11}$ Holloway, P., Pelinovsky, E., and Talipova, T. (2001). Internal tide transformation and oceanic internal solitary waves. In Grimshaw, R., editor, Environmental Stratified Flows, pages 3160. Kluwer.

${ }^{12}$ Leonov, A. I. (1981). The effect of the earth's rotation on the propagation of weak nonlinear surface and internal long oceanic waves. Ann. N.Y. Acad. Sci, 373:150-159.

${ }^{13}$ Ostrovsky, L. (1978). Nonlinear internal waves in a rotating ocean. Oceanology, 18(2):119-125.

${ }^{14}$ Pedlosky, J. (2003). Waves in the Ocean and Atmosphere. Springer.

${ }^{15}$ Renouard, D. and Germain, J.-P. (1994). Experimental study of long nonlinear internal waves in rotating fluid. Ann. Geophysicae, $12: 254-264$.

${ }^{16}$ Stastna, M. F. J., Rowe, K. L., and Subicj, C. (2009). On fully nonlinear, vertically traped wave packets in a stratified fluid on the f-plane. Phys. Fluids, 21:106604.

${ }^{17}$ Vlasenko, V., Sanchez-Garrido, J. C., Stastchuk, N., Lafuente, J. G., and Losado, M. (2009). On fully nonlinear, vertically traped wave packets in a stratified fluid on the f-plane. J. Phys. Ocean., 39:2230-2246. 